Healthcare Infection, 2014, 19, 59-64

$\mathrm{http} / / / \mathrm{dx}$. doi.org/10.1071/HI13034

\title{
ATP bioluminescence to validate the decontamination process of gastrointestinal endoscopes
}

\author{
Geethanie Fernando ${ }^{1} \mathrm{MBBS}, \mathrm{MD}, \mathrm{FRCPA}$ \\ Peter Collignon ${ }^{1,2}$ FRACP, FRCPA, FASM \\ Wendy Beckingham ${ }^{1}$ BHSC (nursing), Grad Cert (Infection Control), MClinical Nurs CICP \\ ${ }^{1}$ The Canberra Hospital, Woden, Canberra, ACT, Australia. \\ ${ }^{2}$ Corresponding author. Email: collignon.peter@gmail.com
}

\begin{abstract}
Introduction: Gastrointestinal endoscopes play an effective diagnostic role in modern medicine. The endoscopes become heavily contaminated with microorganisms during procedures and need careful reprocessing.

Methods: A prospective study was carried out at a gastroenterology hepatology unit to evaluate ATP bioluminescence, measured as relative light units (RLUs), to validate the decontamination processing of endoscopes. Flushes from endoscopes involved in 120 endoscopic procedures at four different stages: pre-patient (before the procedure), post-patient (after the procedure), post-cleaning (after manual cleaning) and post-disinfection were examined by ATP testing and microbiological culture. The hypothetical pass or fail limit of 100 RLUs was set according to previous studies in the literature. When the disinfection process failed, the above process was repeated.

Results: Average RLU readings were: pre-patient: 48; post-patient: 124 052; post-cleaning: 1423; and postdisinfection: 144 . The corresponding culture results were: pre-patient: all negative; post-patient: all positive except for four; post-cleaning: positive except for 26; and post-disinfection: all negative. Although 21 (17\%) of post-disinfection specimens showed failed ATP levels of more than 100 RLUs, when the cleaning and disinfection process was repeated before they were used, all scopes then showed a pass level of less than 100 RLUs.

Conclusions: ATP bioluminescence has the potential to play an important role in the validation process. This process would allow a quick turnaround time following a simple check procedure to be classified as safe in a busy endoscopic unit.
\end{abstract}

Received 1 September 2013, accepted 19 December 2013, published online 11 February 2014

\section{Introduction}

Gastrointestinal endoscopes play an integral part in delivering an effective diagnostic service in modern medicine. When endoscopes are inserted into the gastrointestinal tract, they become heavily contaminated with microorganisms, including potential pathogens. This emphasises the need for careful reprocessing between patients to prevent crossinfection. Inadequately decontaminated endoscopes have been implicated in several healthcare-associated infections (HCAIs) and outbreaks. ${ }^{1,2}$ Endoscopes need to undergo cleaning and appropriate disinfection before re-use. Many guidelines for reprocessing of endoscopes have been produced. ${ }^{3-6}$

Currently in Australia quality reprocessing is checked by performing microbiological cultures according to the Gastroenterological Nurses College of Australia (GENCA) guidelines. $^{7}$ Accordingly, microbiological testing is performed on gastroscopes and colonoscopes 3-monthly with duodenoscopes tested monthly. The initial testing takes up to 1 week to receive a result and with further testing, results take up to 6 weeks (microbiological plus possible TB culture). A major disadvantage is that patients may be potentially exposed to pathogenic microorganisms before the laboratory can issue a result. This may cause increased costs financially, politically and emotionally for the healthcare facility and the patient if a problem is subsequently discovered. Continuity of care can be disrupted if procedures are cancelled because of a lack of equipment when a contaminated piece of equipment needs to be quarantined. Therefore, a more practicable and rapid testing method is desirable.

Adenosine triphosphate (ATP) bioluminescence utilises the light-producing reaction between ATP, luciferin and luciferase to estimate levels of ATP in a sample. The luminometer machine converts the number of photons released into relative light units (RLUs). Adenosine triphosphate is found in organic matter and microorganisms, making estimates of ATP levels a measure of organic soil and contamination. Over the last decade, ATP bioluminescence 


\section{Implications}

- Gastrointestinal endoscopes become heavily contaminated with microorganisms during procedures and need careful reprocessing.

- A prospective study was carried out to evaluate ATP bioluminescence, measured as relative light units (RLUs), to validate the decontamination processing of endoscopes.

- ATP bioluminescence has the potential to play an important role in the validation process.

has become increasingly adopted for monitoring surface cleanliness mainly in the food industry and to a lesser extent in the pharmaceutical industry, and its use is predicted to increase substantially in the near future. ${ }^{8,9}$ This method has been used for measuring levels of organic soil and cleanliness of environmental surfaces and equipment used in hospitals. ${ }^{10-14}$ The ability to provide results within minutes, as opposed to days or weeks for microbiological testing, enables ATP testing to be used as a practical monitoring method. Residual organic matter is an indicator that the surface may be unclean and could provide a potential reservoir that may harbour bacteria, fungi and viruses, increasing the cross-infection risk between patients. Therefore ATP bioluminescence may have a potential role in validating the decontamination process for gastrointestinal endoscopes.

The aim of this study is to evaluate the overall efficacy of standard gastrointestinal (upper and lower) endoscope reprocessing in endoscopy units and to evaluate ATP as a means of assisting in the management of the decontamination process, compared with standard microbiological testing.

\section{Methods}

The study was carried out prospectively at The Canberra Hospital from July to December 2010. The gastroenterology hepatology unit (GEHU) of this hospital performed 4200 endoscopy procedures using five colonoscopes, four gastroscopes and four duodenoscopes from June 2009 to July 2010. The procedures involved banding, clipping of bleeding vessels, biopsies, removal of polyps, dilatation, sphinrectomies and visualisation of the upper or lower gastrointestinal tract. The reprocessing of the used endoscopes was undertaken in the central reprocessing unit (CRU) attached to the GEHU. The processing protocol consists of manual cleaning with an proteolytic enzyme detergent (Aseptic Release Plus, Ecolab Pty Ltd, Sydney) and then by an automated endoscopic reprocessor (Gallay Soluscope 3CC-PAA) using peracetic acid (Gallay Medical \& Scientific, Melbourne, Vic., Australia).

Adenosine triphosphate testing was performed by using 3M 'Clean-Trace' Water-Total ATP swabs (3M, St Paul, MN, USA) with the bioluminometer machine according to the manufacturer's instructions. ${ }^{15}$ Each ATP data point was measured using a single swab, except where some unexpected readings occurred, when additional confirmatory swabs were used. The machine indicates the ATP level in relative light units (RLUs) in less than 2 min. Firstly, external quality controls were checked by using the known ATP positive and negative control products which were commercially available. The internal quality controls were checked by using Brain Heart Broths (Merck KGaA, Darmstadt, Germany) at different dilutions with sterile distilled water. During the study period, quality-control testing was done using the commercially available known ATP positive and negative controls on a monthly basis. The internal positive controls ( $1: 10$ dilution of sterile Brain Heart Broth) and the negative controls (sterile distilled water) were done weekly.

The ATP levels were tested against different organisms such as Staphylococcus aureus ATCC 29213, Enterococcus faecalis ATCC 29212, Escherichia coli ATCC 25922, Pseudomonas aeruginosa ATCC 27853, Candida albicans ATCC 10231 and Mycobacterium fortuitum ATCC 6841. From each organism a $0.5 \mathrm{McF}$ arland standard was made up with sterile Brain Heart Broth (Merck KGaA) and then diluted eight times with $1: 10$ dilution at each step with sterile distilled water. Each tube was tested for ATP level and at the same time cultured on to Horse Blood agar (bioMérieux, Marcy L'Etoile, France) and incubated at $30^{\circ} \mathrm{C}$ for 24 to $48 \mathrm{~h}$.

During the study period we examined 120 endoscopes (59 colonoscopes, 50 gastroscopes and 11 duodenoscopes). The testing was performed at four different stages: before inserting the endoscope into the patient (pre-patient: step 1), just after the procedure (post-patient: step 2), after the manual cleaning with the detergent (post-cleaning: step 3 ) and finally after completion of the disinfection process (post-disinfection: step 4). At each step the endoscope biopsy and suction channels were flushed with $20 \mathrm{~mL}$ of sterile $0.9 \%$ saline. The flush fluid was collected aseptically in the CRU and sent to the microbiology laboratory without delay. If a delay was expected, the sample was stored at $4^{\circ} \mathrm{C}$.

In the laboratory, the ATP testing was performed using the 'Clean-Trace' Water-Total ATP swabs (3M) and the RLU value recorded. Each flush fluid sample was inoculated onto blood agar (MacConkey) and incubated at $30^{\circ} \mathrm{C}$ and $35^{\circ} \mathrm{C}$ respectively for 7 days. The plates were examined daily for growth. Any growth was quantified and identified to species level using basic manual microbiological techniques. ${ }^{16,17}$

The hypothetical pass or fail limit of 100 RLUs (pass $\leq 100$, fail $>101$ ) was set according to the results of Hansen et al. ${ }^{18}$ When the post-disinfection fluid ATP level was >101 RLUs, the CRU was informed and requested to repeat the disinfection process and rechecked the flush fluid for ATP and microbiology culture. The failed specimens were tested for proteins ${ }^{19}$ to exclude biological contamination.

\section{Statistical analyses}

Adjusted R-squared values from a linear regression were reported to indicate the fraction of the variance of the log ATP (RLU) explained by the log of bacterial concentrations for 
different organisms. The Kruskal-Wallis test was used to test for difference in means across steps (pre-patient, post-patient, post-cleaning and post-disinfection) for the 120 endoscopes. The Wilcoxon signed-rank sum test was used to test for differences between the individual steps.

\section{Results}

A 0.5 McFarland concentration of each of the organisms S. aureus, E. faecalis, E. coli, P. aeruginosa, C. albicans and M. fortuitum was diluted 1:10 for eight times with sterile distilled water and at each step the ATP level was measured.

Fig. 1 shows the pattern of ATP levels with the various organisms at different concentrations. There was a good relationship with increased numbers of individual organisms and measured ATP levels. While there was the same

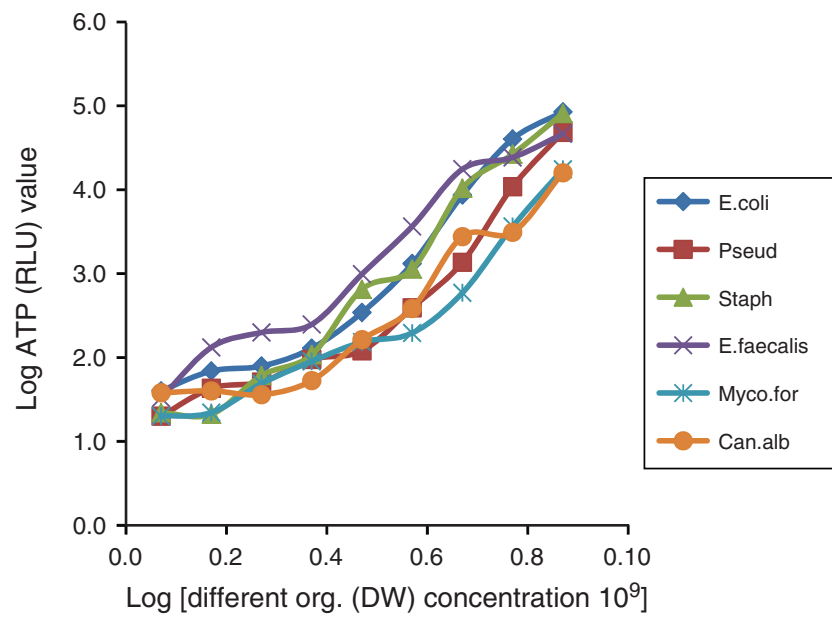

Fig. 1. Log ATP levels for organisms at different dilutions with sterile distilled water (DW). E.coli, Escherichia coli ATCC 25922 (adjusted R²: 0.9251); Pseud, Pseudomonas aeruginosa ATCC (adjusted $\mathrm{R}^{2}$ : 0.8977); Staph, Staphylococcus aureus ATCC 29213 (adjusted R²: 0.9678); E.faecalis, Enterococcus faecalis ATCC 29212 (adjusted $\mathrm{R}^{2}$ : 0.9745); Myco. for, Mycobacterium fortuitum ATCC 6841 (adjusted $\mathrm{R}^{2}$ : 0.9130); Can. alb, Candida albicans ATCC 10231 (adjusted $\mathrm{R}^{2}$ : 0.8844). increasing ATP/numbers relationship with all microorganisms, there were variations with different organisms (Table 1).

Table 1 shows the in vitro testing of ATP of the organisms in different dilutions. At the final dilution step ( $\log$ concentration 0.7) the colony count was zero for all six organisms although the ATP level varied from 20 to 40 RLUs. At the 2.7 Log dilution step E. coli and $S$. aureus show 5 and $9 \mathrm{CFU}$, although the corresponding ATP level is below the 100 RLUs which is the hypothetical cut-off.

Fig. 2 shows how the ATP level varied from the pre-patient step to the post-disinfection step. All the post-patient and the majority of post-cleaning specimens gave high readings (>100 RLUs), which is the failed level and as expected. Across steps there was a significant difference in means of $\log 10$ (RLU) [pre-patient: 1.53 (1.47-1.58), post-patient: 4.54 (4.38-4.69), post-cleaning: $2.66(2.55-2.77)$ and postdisinfection: $1.70(1.60-1.79), P<0.0001]$. There were statistically significant differences between individual steps in $\log 10$ (RLU); pre-patient to post-patient: -3.01 ( -3.18 to $-2.84)$, post-patient to post-cleaning: $1.88(1.75-2.02)$, postcleaning to post-disinfection: $0.96(0.87-1.05), P<0.0001$ in all three instances. Although the pre-patient and postdisinfection level specimens were expected to be below the pass level ( $\leq 100$ RLUs), 21 specimens $(17 \%)$ did not meet this criterion (Table 2).

All cultures were positive at the post-patient step except for four gastroscope specimens, although in these four cases the ATP levels were above 1000 RLUs. Of 50 post-cleaning gastroscope specimens, 26 were culture-negative. In these, the ATP level was between 101 and 1000 RLUs. The cultures were negative at both pre-patient and post-disinfection steps in all colonoscopes, gastroscopes and duodenoscopes.

Thirty-three of these endoscopies were performed to visualise the upper or lower gastrointestinal tract without performing invasive procedures. Biopsies and polyps were removed in 42 and 15 occasions respectively, while both were performed on 7 occasions. Both gastroscopy and colonoscopy was performed using the same endoscope on 14 occasions.

Table 1. In vitro testing of different organisms in different concentrations (ATP value (RLU) versus colony forming units/ $10 \mu \mathrm{L}$ solution (CFU))

Ecoli, Escherichia coli ATCC 25922; Pseud, Pseudomonas aeruginosa ATCC; Staph, Staphylococcus aureus-ATCC 29213; Ent fae, Enterococcus faecalis ATCC 29212; Myco for, Mycobacterium fortuitum ATCC 6841; Can alb, Candida albicans ATCC 10231

\begin{tabular}{llrrrrrr}
\hline Log con & Measurement & Ecoli & Pseud & Staph & Ent fae & Myco for & Can alb \\
\hline 5.7 & RLU & 1316 & 392 & 1137 & 3660 & 196 & 384 \\
4.7 & CFU & 300 & 500 & 300 & 500 & 500 & 100 \\
& RLU & 344 & 120 & 651 & 986 & 153 & 163 \\
3.7 & CFU & 92 & 150 & 162 & 150 & 100 & 5 \\
& RLU & 129 & 95 & 108 & 248 & 90 & 53 \\
2.7 & CFU & 37 & 21 & 19 & 14 & 15 & 0 \\
& RLU & 79 & 51 & 61 & 199 & 49 & 36 \\
1.7 & CFU & 5 & 1 & 9 & 2 & 1 & 0 \\
& RLU & 69 & 43 & 21 & 132 & 1 & 0 \\
0.7 & CFU & 1 & 0 & 3 & 33 & 20 & 0 \\
& RLU & 40 & 20 & 22 & 0 & 0 & 38 \\
& CFU & 0 & 0 & 0 & & 0 \\
\hline
\end{tabular}




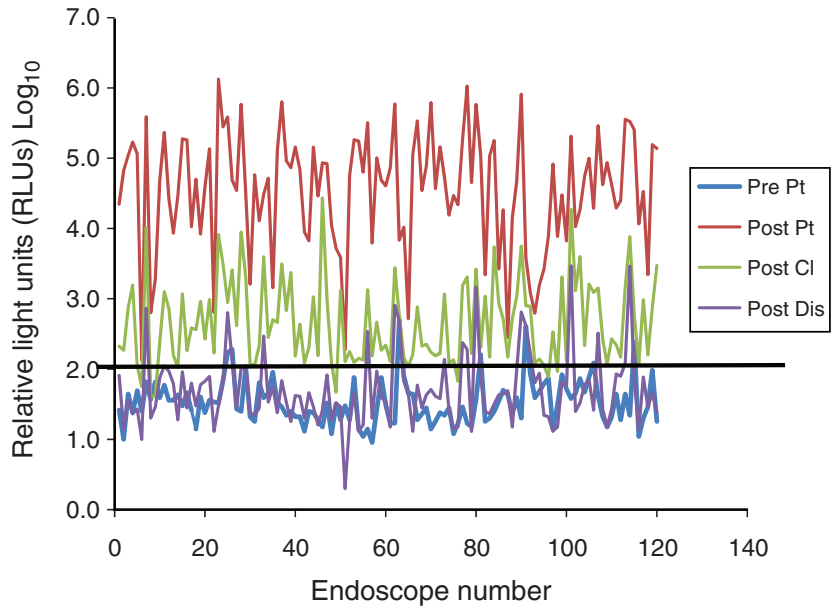

Fig. 2. Measured ATP value at pre-patient (pre pt), post-patient (post pt), pos- cleaning (post cl), post-disinfection (post dis) step for 120 endoscopes.

Table 2. Scopes with high ATP RLU ( $>100$ RLUs) values

\begin{tabular}{llccc}
\hline No. & Scope identity & Pre-patient & Post-disinfection & Repeat ATP \\
\hline 1 & Gastroscope 112 & 66 & 733 & 53 \\
2 & Gastroscope 111 & 59 & 111 & Not tested \\
3 & Gastroscope 112 & 171 & 633 & 24 \\
4 & Colonoscope 207 & 190 & 134 & 23 \\
5 & Gastroscope 111 & 25 & 103 & Not tested \\
6 & Ccolonoscope 206 & 109 & 111 & Not tested \\
7 & Gastroscope 111 & 39 & 294 & 32 \\
8 & Gastroscope 112 & 14 & 341 & 53 \\
9 & Gastroscope 112 & 17 & 809 & 13 \\
10 & colonoscope 205 & 504 & 485 & 15 \\
11 & Colonoscope 206 & 22 & 137 & 16 \\
12 & Gastroscope 110 & 29 & 237 & 27 \\
13 & Gastroscope 111 & 17 & 191 & 17 \\
14 & Gastroscope 110 & 37 & 1463 & 32 \\
15 & Gastroscope 111 & 20 & 648 & 59 \\
16 & Colonoscope 206 & 406 & 414 & 33 \\
17 & Duodenoscope 308 & 87 & 171 & 25 \\
18 & Colonoscope 206 & 38 & 2930 & 24 \\
19 & Gastroscope 110 & 41 & 324 & 47 \\
20 & Gastroscope 111 & 44 & 123 & 21 \\
21 & Gastroscope 111 & 22 & 2908 & 30 \\
\hline
\end{tabular}

Fig. 3 shows the average ATP Log values for each step during the process. There was a $\log 2$ reduction of the ATP level from the post-patient step to the post-cleaning step and suggests satisfactory manual cleaning. At the postdisinfection step, the ATP levels further reduced by Log 1 .

Table 2 shows the scopes which gave the failed ATP level at the post-disinfection step. Out of 120 scopes, 21 (17\%) gave a failed ATP level (>101 RLUs) at the post-disinfection step. For these corresponding specimens the cultures were negative and protein levels were undetected. There were no significant invasive procedures done using these scopes. The colonoscopes which gave higher ATP readings (>100 RLUs) at the pre-patient step ended up also as high at the post-patient

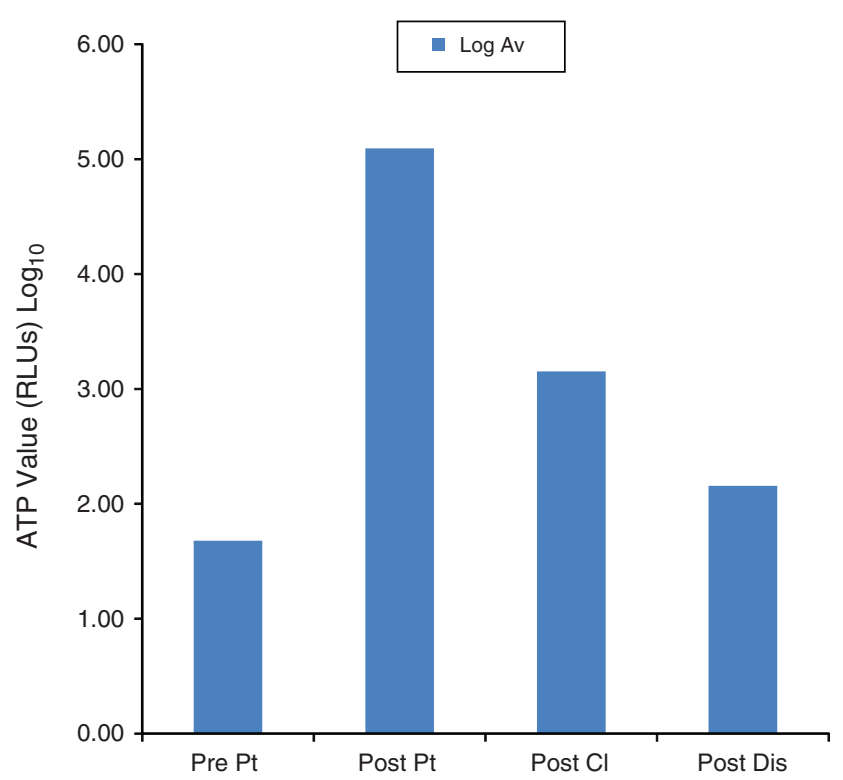

Fig. 3. Comparison of average ATP $\left(\log _{10}\right)$ values for pre-patient (pre pt), post-patient (post pt), post-cleaning (post cl) and post-disinfection (post dis).

Table 3. Fail number to total number of scopes at different hypothetical ATP cut-off levels

Pre col, pre-patient colonoscope; pre gas, pre-patient gastroscope; pre duo, pre-patient duodenoscope; post col, post-patient colonoscope; post gas, postpatient gastroscope; post duo, post-patient duodenoscope

\begin{tabular}{lcccccc}
\hline $\begin{array}{l}\text { ATP cut- } \\
\text { off (RLU) }\end{array}$ & \multicolumn{3}{c}{$\begin{array}{c}\text { Fail no./total } \\
\text { (pre-patient) }\end{array}$} & \multicolumn{3}{c}{$\begin{array}{c}\text { Fail no./total } \\
\text { (post-disinfection) }\end{array}$} \\
& Pre col & Pre gas & Pre duo & Post col & Post gas & Post duo \\
\hline$>50$ & $9 / 59$ & $14 / 50$ & $3 / 11$ & $15 / 59$ & $32 / 50$ & $3 / 11$ \\
$>100$ & $6 / 59$ & $1 / 50$ & $1 / 11$ & $6 / 59$ & $12 / 50$ & $1 / 11$ \\
$>300$ & $2 / 59$ & $0 / 50$ & $0 / 11$ & $3 / 59$ & $8 / 50$ & $0 / 11$ \\
$>500$ & $1 / 59$ & $0 / 50$ & $0 / 11$ & $1 / 59$ & $6 / 50$ & $0 / 11$ \\
\hline
\end{tabular}

step. However, the gastroscopes which started as low ATP $(<100$ RLUs) at the pre-patient step ended up with high (failed) ATP levels at the post-patient step. After repeating the automated machine disinfection cycle for these scopes, all the specimens except for three which were not tested showed low ATP $(<100$ RLUs) levels.

Table 3 shows how the hypothetical ATP cut-off levels varied for pre-patient and post-disinfection steps in all the scopes. The hypothetical cut-off level at 50 gave the maximum failure rate compared with least failure rate at the 500 cut-off level.

\section{Discussion}

Gastrointestinal endoscopy is a common clinical procedure in modern medicine. The risk of transmitting infections via this procedure depends on factors such as exposure of the endoscope to microorganisms or biological body fluids, cleaning and disinfection procedures, and the design of the instrument. Because endoscopes are made of heat-sensitive 
materials, they are routinely decontaminated by high-level disinfection rather than heat. Although the validity of this process can be checked by routine microbiological cultures, the usefulness of this is limited by the time factor and inability to perform testing with each procedure. The ATP bioluminescence procedure overcomes the above issues and therefore appears suitable for further investigation.

Fig. 1 shows the variations in levels of ATP production with various organisms. When organisms were present in low numbers, they were often not detected by ATP testing. Other investigators have found this and that ATP concentration varies over the growth cycle, population ages and even on the culturing environment. ${ }^{20}$

Effective cleaning is a vital part of instrument reprocessing. Visual assessment has limitations and is difficult to apply to an endoscope because of the complexity of the instrument. In this study the use of ATP bioluminescence showed a Log 2 reduction from the post-patient step to the post-cleaning step. This is an excellent objective measure of confirming the reduction in contamination. The final step, the postdisinfection step, showed a further log reduction. While it should be noted that ATP measurements have inherent variability, ATP levels still separate 'clean' and 'dirty' areas well with substantial differences in the underlying ATP soil level. ${ }^{20}$

Out of 120 scopes, 21 (17\%) gave failed ATP levels (>101 RLUs) at the post-disinfection step even though the cultures were negative and proteins were not detected. It was interesting to note that the 14 gastroscopes, except for one, started as low ATP $(<100$ RLUs $)$ at the pre-patient step and ended as high ATP ( $>100$ RLUs) at the post-disinfection step, in contrast to the six colonoscopes, except for two, which started and ended as high ATP ( $>100$ RLUs) at both the prepatient and post-disinfection steps. This difference was not related to the endoscopy procedure as the scopes used for invasive procedures gave low ATP readings and vice versa. This may be attributed to the difference in the contents of fluid in the upper and lower gastrointestinal tract including the $\mathrm{pH}$. The manufacturer claimed that the $3 \mathrm{M}$ 'Clean-Trace' Water-Total ATP swabs coupled with the bioluminometer machine is not affected by the $\mathrm{pH}$ within the range of 3-10. However all the corresponding repeat specimens (except three which were not checked) showed low ATP $(<100$ RLUs), which means that some unidentified factor (possibly a small amount of residual protein or other substance that then decayed over a period of time) was cleared with the repeat disinfection cycle. More investigations such as micro scanning of the fluid or perhaps molecular testing may be required to find an explanation for the lowering of ATP after the repeat disinfection cycle.

The hypothetical pass or fail limit of 100 RLUs was set according to the results of Hansen et al. ${ }^{18}$ In our study 21 of 120 instruments (17\%) measured ATP bioluminescence was above the pass or fail limit on instruments after they were initially processed but when these were reprocessed all except for three were below 100 RLUs $(P<0.001)$. Different researchers used different levels of cut-off depending on either their personal preference, past experience or the type of ATP device or machine. Literature reviewed identified a limit of 500 RLUs using Uni-Lite (Biotrace International), ${ }^{21} 100$ RLUs using an ultra-snap sampling device from Hygiena, ${ }^{22}$ 100 RLUs using Uni-Lite Xcel (Biotrace International) ${ }^{23}$ and between 30 to 100 RLUs using Lumitester PD 10 (Scil Diagnostics). ${ }^{24}$ Some recent smaller studies have been performed, ${ }^{13,14}$ but do not use always 'in-use' endoscopes. Fushimi et al. ${ }^{14}$ confirm that using our cut-off value of 100 RLUs is appropriate. In their study before cleaning, ATP values were 10417 RLUs from the exterior endoscope surface and 30281 RLUs from the suction or accessory channel rinsates. After cleaning, these ATP values were decreased to 82 RLUs and 104 RLUs.

We assessed four different hypothetical cut-off levels (Table 3). The cut-off level of $>500$ RLUs gave the minimum failure rate. But this cut-off is not acceptable due to the fact that we experienced a growth of a considerable number of organisms at this level (Table 1). The cut-off level of 50 RLUs is theoretically very good as there were no organisms grown (Table 1), but the 'failure' rate was also maximal and would then be an indication for more reprocessing of the endoscopes, which is not practical in a busy endoscopic clinic. According to the manufacturer's instructions, the $3 \mathrm{M}$ 'Clean-Trace' bioluminometer machine has an inherent ATP reading of 20 to 25 RLUs for negative specimens. ${ }^{15}$ Therefore the $>100$ RLUs cut-off was chosen to be more logical and practical.

At the beginning of the study the $3 \mathrm{M}$ 'Clean-Trace' WaterTotal ATP swabs failed to show the correct positive ATP value (>1000 RLUs) with the commercially-available known positive ATP controls. However, the negative controls $(<20-25$ RLUs) were within the standard limits. Later this error was rectified with a new batch of swabs which indicated the results within the positive and negative control limits according to the manufacturer's instructions. Sterile Brain Heart Broth, a 1:10 dilution of sterile Brain Heart Broth (diluted with sterile distilled water) and sterile distilled water were set as high positive ( $>1000$ RLUs), low positive (200-300 RLUs), and negative (20-25 RLUs) internal controls as an added quality-assurance measure. During the study close monitoring of quality-assurance of the swabs and the machine was set in order to obtain reliable ATP values.

In conclusion, our results showed that measurements of ATP bioluminescence with a hand-held device may have an important role to play to help easily and quickly validate the decontamination process of gastrointestinal endoscopes. As the results are available within $2 \mathrm{~min}$, this makes it possible to do the test before each procedure in a busy endoscope clinic and should allow the task to become routine. Further studies are required to allow closer quality-control settings for accurate results.

\section{Conflicts of interest}

The authors have no conflicts to declare. 


\section{Funding}

The ATP bioluminometer machine was funded by the Private Practice Fund at The Canberra Hospital, Woden, ACT. The first 500 'Clean-Trace' Water-Total ATP swabs were supplied free of charge by $3 \mathrm{M}$ Australia.

\section{Acknowledgements}

We would like to thank Grace Joshy for her assistance with the statistical analysis and the sterilisation staff based at The Canberra Hospital for their helpfulness.

\section{References}

1. Spach D, Silverstain F, Stamm W. Transmission of Infection by Gastrointestinal Endoscopy and Bronchoscopy. Ann Intern Med 1993; 118: 117-28. doi:10.7326/0003-4819-118-2-199301150-00008

2. Bronowicki J, Venard V, Botte C, Monhoven N, Gastin I, Chone L, et al. Patient to patient transmission of hepatitis $\mathrm{C}$ virus during colonoscopy. $N$ Engl J Med 1997; 337: 237-40. doi:10.1056/ NEJM199707243370404

3. British Society of Gastroenterology Endoscopy Committee. BSG Guidelines for Decontamination of Equipment for Gastrointestinal Endoscopy. London: British Society of Gastroenterology; 2003. Available from: http://www.bsg.org.uk/clinical-guidelines/endoscopy/ guidelines-for-decontamination-of-equipment-for-gastrointestinalendoscopy.html [verified January 2014].

4. Nelson DB, Jarvis WR, Rutala WA, Foxx-Orenstein AE, Isenberg G, Dash GR, et al. Multi-society guideline for reprocessing flexible gastrointestinal endoscopes. Infect Control Hosp Epidemiol 2003; 24: $532-7$.

5. European Society of Gastrointestinal Endoscopy. ESGE-ESGENA Guideline: Cleaning and Disinfection in Gastrointestinal Endoscopy Update. Munich, Germany: ESGE; 2008. Available from: http://www. esge.com/esge-guidelines.html [verified January 2014].

6. Centers for Disease Control and Prevention. Guideline for Disinfection and Sterilization in Healthcare Facilities. Atlanta, GA: CDC; 2008. Available from: http://www.cdc.gov/hicpac/disinfection_steriliza tion/3_0disinfectequipment.html [verified January 2014].

7. Queensland Health. Endoscope Reprocessing. The State of Queensland (Queensland Health); 2012. Available from: http://www. health.qld.gov.au/EndoscopeReprocessing/default.asp [verified January 2014].

8. Aycicek H, Oguz U, Karci K. Comparison of results of ATP bioluminescence and traditional hygiene swabbing methods for the determination of surface cleanliness at a hospital kitchen. Int J Hyg Environ Health 2006; 209(2): 203-6. doi:10.1016/j.ijheh.2005.09.007

9. Jimenez L. Adenosine triphosphate bioluminescence analysis for rapid screening of microbial contamination in non-sterile pharmaceutical samples. PDA J Pharm Sci Technol 2004; 58(3): 159-68.

10. Lewis T, Griffith C, Gallo M, Weinbren M. A modified ATP bench mark for evaluating the cleaning of some hospital environmental surfaces. J Hosp Infect 2008; 69: 156-63. doi:10.1016/j.jhin.2008. 03.013

11. Andersen BM, Rasch M, Kvist J, Tollefsen T, Lukkassen R, Sandvik L, et al. Floor cleaning: effect on bacteria and organic materials in hospital rooms. J Hosp Infect 2009; 71(1): 57-65. doi:10.1016/j.jhin.2008. 09.014

12. Smith PW, Sayes H, Hewlett A, Gibbs SG, Rupp ME. A study of three methods for assessment of hospital environmental cleaning. Healthc Infect 2013; 18: 80-5. doi:10.1071/HI13001

13. Alfa MJ, Fatima I, Olson N. Validation of adenosine triphosphate to audit manual cleaning of flexible endoscope channels. Am J Infect Control 2013; 41: 245-8. doi:10.1016/j.ajic.2012.03.018

14. Fushimi R, Takashina M, Yoshikawa H, Kobayashi H, Okubo T, Nakata $\mathrm{S}$, et al. Comparison of adenosine triphosphate, microbiological load, and residual protein as indicators for assessing the cleanliness of flexible gastrointestinal endoscopes. Am J Infect Control 2013; 41: 161-4. doi:10.1016/j.ajic.2012.02.030

15. 3M. 'Clean-Trace' Water-Total ATP swabs product information. St Paul, MN: 3M. Available from: http://solutions.3m.com/wps/portal/ 3M/en_US/Microbiology/FoodSafety/product-information/productcatalog/?PC_Z7_RJH9U523003DC023S7P92O3O87000000_nid= QQ3B1KTHFPbeJ9C52DTHJWgl [verified January 2014].

16. Winn WC, Allen SD, Janda WM, Koneman EW, Procop GW, Schrenckenberger PC, et al. Gram positive cocci, gram positive bacilli. In: Koneman EW, ed. Koneman's Color Atlas and Textbook of Diagnostic Microbiology. 6th edn. Philadelphia, PA: Lippincott Williams \& Wilkins; 2006: pp. 674-840.

17. Winn WC, Allen SD, Janda WM, Koneman EW, Procop GW, Schrenckenberger PC, et al. The Enterobacteriaceae, The Nonfermentative Gram-negative Bacilli. In: Koneman EW, ed. Koneman's Color Atlas and Textbook of Diagnostic Microbiology. 6th edn. Philadelphia, PA: Lippincott Williams \& Wilkins; 2006: pp. 213-316.

18. Hansen D, Hilgenhoner M, Popp W. ATP bioluminescence - for kitchen hygiene and cleaning control of surgical instruments. Intern $J$ Infect Control 2008; 4: 1-4. doi:10.3396/ijic.V4i1.010.08

19. Chemiluminecent Miroparticle Immunoassay for Qualitative Detection of Urine Proteins - Abbott ARCHITEC c8000 Chemistry analyser. Available from: http://www.abbottdiagnostic.com [verified January 2014].

20. Shama G, Malik DJ. The uses and abuse of rapid bioluminescencebased ATP assays. Int J Hyg Environ Health 2013; 216(2): 115-25. doi:10.1016/j.ijheh.2012.03.009

21. Obee P, Griffith C, Cooper R, Cooke R, Lewis M. Real-time monitoring in managing the decontamination of flexible gastrointestinal endoscopes. Am J Infect Control 2005; 33: 202-6. doi:10.1016/j.ajic.2004.07.008

22. Heathcote R, Stadelmann B. Measuring of ATP bioluminescence as a means of assessing washer disinfector performance and potentially as a means of validating the decontamination process. Healthc Infect 2009; 14: 147-51. doi:10.1071/HI09019

23. Davidson CA, Griffith CJ, Peters AC, Fielding LM. Evaluation of two methods for monitoring surface cleanliness-ATP bioluminescence and traditional hygiene swabbing. Luminescence 1999; 14: 33-8. doi:10.1002/(SICI)1522-7243(199901/02)14:1<33::AID-BIO514> 3.0.CO;2-I

24. Dorothea H, Benner D, Hilgenhoner M, Leisebein T, Brauksiepe A, Popp W. ATP measurement as method to monitor the quality of reprocessing flexible endoscopes. Ger Med Sci 2004; 2: 1-5. 